\title{
Proteins and proteomics: life on the surface
}

\author{
Nathan Blow
}

\author{
Surface plasmon resonance sensing has entered the next phase of development as researchers advance \\ array-based applications using the technique. Could these new approaches change the way scientists \\ explore protein interactions?
}

In his laboratory at the University of California in Irvine, chemistry professor Robert Corn has spent the better part of twenty years developing and advancing surface plasmon resonance (SPR) sensing techniques to explore biomolecule interactions. "SPR is naturally suited to detecting protein binding or finding protein biomarkers because you do not need a label to perform the experiments," he says.

But in today's high-throughput proteomics world in which researchers are interested in studying hundreds, if not thousands, of proteins and their interactions with other biomolecules at once, even the draw of working label-free is not enough to entice most scientists to SPR approaches, which traditionally tests only a few purified protein samples at a time. "To do a proper proteomics study you want to be able to study multiple interactions simultaneously," notes Corn, which is why his group and others are working to change the face of SPR techniques in the hopes of spurring the technology into wider use.

\section{Humble beginnings}

"SPR sensing is really dirt-trivial to perform," says University of Washington chemistry professor Charles Campbell. In a typical SPR sensing experiment one partner of a potential interacting pair of biomolecules is immobilized on a goldcoated glass slide and a solution containing the other partner is flowed across the slide's surface. By shining light up through the glass and onto the gold surface at specific angles and wavelengths, researchers can measure slight changes in the optical refractivity of the gold when a biomolecule is attached to the surface. And that optical

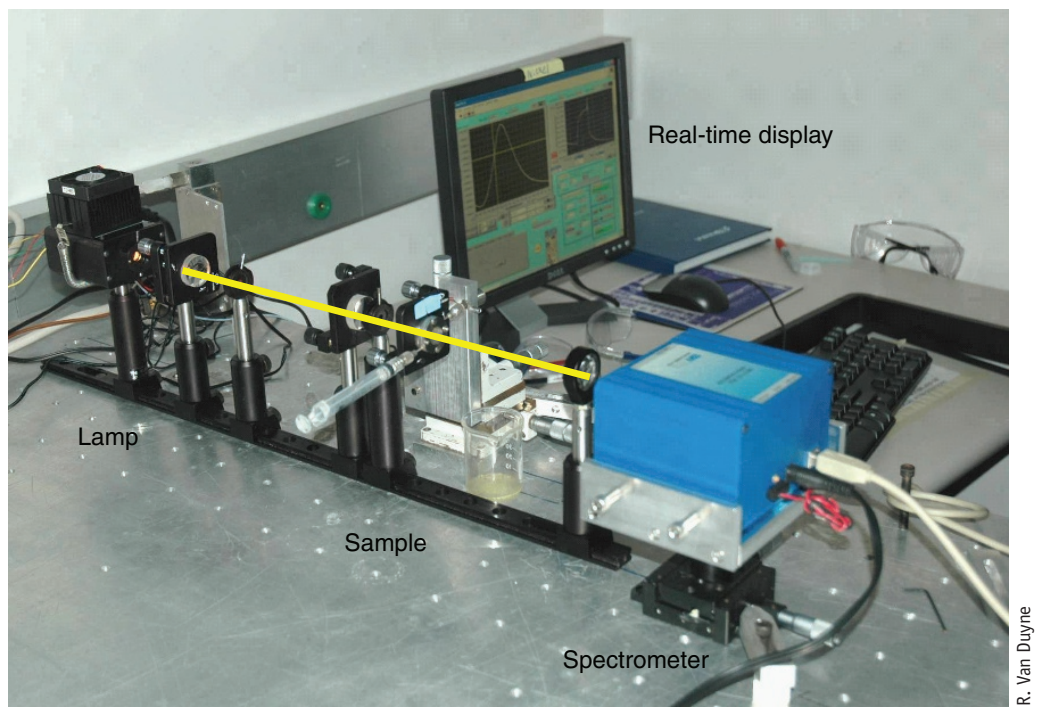

Experimental system for performing high-resolution LSPR sensing.

refractivity again changes when the immobilized partner binds another biomolecule from the solution.

SPR analysis is exquisitely sensitive to monitoring such binding interactions. "This is one of the huge positive features of SPR because if you do the measurement right, you are not limited to biomolecule binding; you can see small molecular binding events as well," explains Richard Van Duyne, a chemistry professor at Northwestern University. Van Duyne says that some current SPR techniques are sensitive to changes in structure or binding events approaching the single molecule level of detection.

Instruments based on SPR biosensing were developed in the late 1980s and became commercially available in the early 1990s. One of the first, and still most common, systems is the Biacore platform developed by Pharmacia and now offered through GE Healthcare. The Biacore approach to SPR sensing makes use of a flow cell to deliver biomolecules and a sensor chip with a gold surface that is coated with a layer of hydrogel to immobilize proteins. "Once in water, the hydrogel used in the surface chemistry extends about 100-200 nanometers away from the metal surface, so a receptor can be immobilized throughout that depth, giving a greater number of receptors than you could get if they were closely packed in a monolayer," says Campbell, describing an advance that has improved SPR detection sensitivity by taking advantage of the fact that binding events up to 200 nanometers from the gold surface can induce detectable changes in refractivity.

Following in the footsteps of the Biacore platform, several developers_-including 


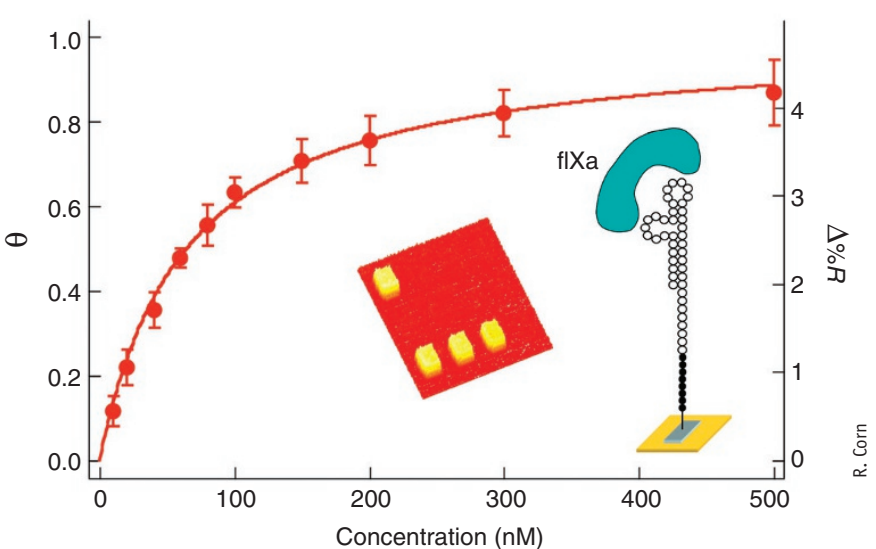

Plot of adsorption of a serine protease to an aptamer array at different concentrations using SPR imaging. Inset on left is a three-dimensional SPR imaging difference image, and the right inset is a schematic of aptamer attachment on array. $\theta$, relative surface coverage; and $R$, reflectivity.

GWC Technologies, Fujifilm Life Science, SensiQ and Bio-Rad-are designing new SPR sensing instruments and approaches for a variety of research applications.

\section{An array of hope}

"I think plasmon resonance imaging has really come into its own," says Corn. SPR imaging, or SPR microscopy as some call it, is now being used to detect protein interactions on a flat array format. Unlike fluorescent array technology in which a label is used and a change in fluorescence signal intensity is measured when a binding event occurs, SPR imaging provides a quantitative, label-free way to explore biomolecule interactions.

According to Campbell, SPR imaging also provides an extremely efficient way to simultaneously get SPR curves for a great number of spots on a gold surface. "People have done it with up to 2,000 spots, and you get one-second time resolution," he says. Another advantage of using an array over a traditional SPR instrument, which might not be obvious at first, is the smaller amount of reagents necessary because the immobilized spots on the surface of these arrays use very small amounts of material and the solutions injected into flow chambers probe many spots simultaneously, also increasing efficiency.

SPR imaging applications are being reported with increasing frequency, with researchers creating carbohydrate arrays, peptide arrays, DNA arrays and antibody arrays to explore protein binding. Corn's group is now working on aptamer arrays and recently showed that it was possible to sense the binding of proteases to aptamers using SPR. "Antibodies are rather fragile, but aptamers are a little more robust," says Corn. And GWC Technologies, a company cofounded by Corn in 1997, has developed an SPR imaging system called SPRimager II along with arrays that can be robotically or manually spotted by researchers.

One SPR imaging convert is proteomics researcher Joshua LaBaer, who is also director of the newly created Virginia G. Piper Center for Personalized Diagnostics. "There is a Biacore instrument in every academic department, but the number of users is small, and I think that has to do with not wanting to purify proteins beforehand," he suspects. For LaBaer and others who are interested in looking at hundreds of different interactions, purifying that many proteins before testing poses a tremendous

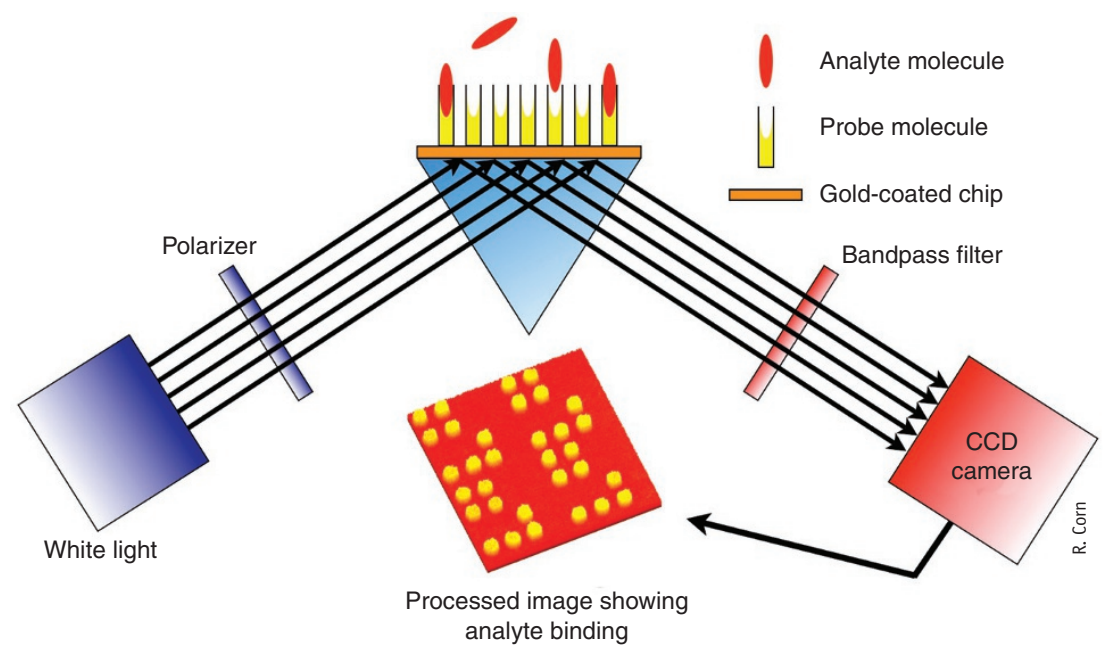

Diagram of SPR imaging setup. CCD, charge-coupled device. bottleneck in their experiments. But some SPR imaging platforms provide a possible solution to the problem.

LaBaer's group, along with three other labs, were beta testers for the Kx5 platform developed by a company called Plexera. The Kx5 platform, much like Corn's approach with the aptamer arrays, uses an array format that can be printed with biomolecules before processing, potentially eliminating the need to purify proteins before an SPR sensing experiment by allowing researchers to express their proteins of interest in situ. LaBaer's group has used their instrument to explore over 400 different interactions at the same time while obtaining both kinetic and affinity information.

One year ago Plexera went out of business, falling victim to what LaBaer thinks is a harsh reality when it comes to SPR sensing technology. "People have not yet seen the potential of using SPR," he says. Today his group keeps their $\mathrm{Kx} 5$ platform working while crossing their fingers in the hopes that something else comes along to fill the void.

Bio-Rad is hoping they can help change the way researchers perceive SPR sensing with their newly released ProteOn XPR36 protein interaction array system. "We started by looking for a simple solution that would expand upon the traditional ability of SPR," says Cathy Mainini, a senior product manager for proteomics at Bio-Rad. Unlike the Plexera or GWC Technologies approach using pre-spotted arrays, the ProteOn creates a $6 \times 6$ grid of crossing microfluidics channels on a gold surface coated with alginate to generate 36 crossing points where SPR sensing data can be collected. According to Mainini, 


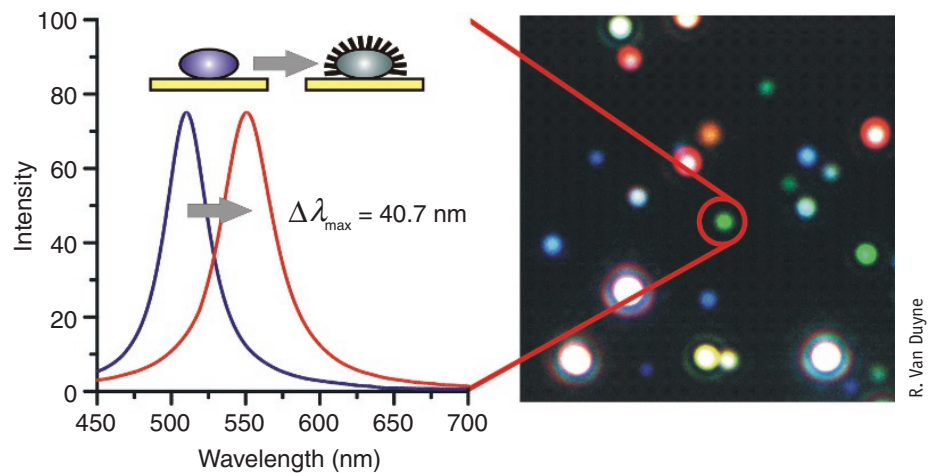

In LSPR, nanoparticles shift the wavelength of plasmon resonance, creating the potential for singlebiomolecule interaction studies. $\lambda_{\text {max }}$ wavelength of maximum absorbance.

the system provides a unique format that allows researchers to test multiple conditions, such as $\mathrm{pH}$ and salt concentrations, on multiple samples to determine optimum conditions for initial protein immobilization to the metal surface as well as protein interaction assays.

There are also two Biacore systems now using microfluidic array approaches in their design. The Biacore A100 takes advantage of four independent flow cells, each capable of immobilizing up to five proteins, while the sensor chip in the Flexchip system has 400 spots for the immobilization and testing of biomolecules against a specific sample.

Many researchers suggest the range of SPR sensing applications is still hampered by the tricky issue of surface functionalization especially for array applications. "In many applications, you are covering the whole area with the surface chemistry that you want, but for arrays you have 50-200 [micrometer] spots with your desired surface chemistry and then you have to have the area in between the spots properly separated," explains Campbell. Corn says surface chemistry that gives more surface specificity and sensitivity will continue to be an important area of SPR development in the coming years. "We have done a great job with the surface chemistry for nucleic acids, but not so much with other biomolecules such as proteases," he says.

\section{Sensitive situations}

Advances in SPR imaging are allowing researchers to look at large numbers of protein-protein interactions in the nanomolar range. To go even lower, requires slightly different ways of SPR sensing. "Nanoparticleenhanced SPR imaging takes us down into the femtomolar range," says Corn.
One of the first demonstrations of the use of nanoparticles for SPR biosensing was performed by Van Duyne and his colleagues, a technique he coined localized SPR (LSPR) ${ }^{1}$. "One of the themes of our work has been how to push the sensitivity of this technique and determine just what the physical boundaries are," he says. For Van Duyne the 'holy grail' of SPR is the ability to measure single biomolecule binding events, both inside and outside the cell.

LSPR measures the wavelength shift in the plasmon resonance peak of a nanoparticle. This differs from traditional SPR sensing in which intensity is measured, meaning that a reference channel or spot is required to determine any intensity changes, which is not the case for LSPR. "The wavelength shift is an absolute measure, all the information comes from measuring this shift," says Van Duyne making it theoretically possible to perform singleparticle interaction experiments.

Not only is the LSPR shift absolute, "the shift is absolutely linear with respect to the number of molecules bound," Van Duyne says, which makes it possible to generate predictions for single-molecule binding events.

Most of Van Duyne's work with LSPR up to now has been using arrays of nanoparticles to test interactions. While proving effective, similar to SPR imaging on flat arrays, LSPR is not immune to the challenges of finding good surface chemistry for immobilization of biomolecules to the surface of the particles. For example, Van Duyne and colleagues recently created a fully functional prostate-specific antigen sensor based on LSPR. According to Van Duyne, everything works, with one minor hitch at the moment: their LSPR sensor is 10,000 times less sen- 

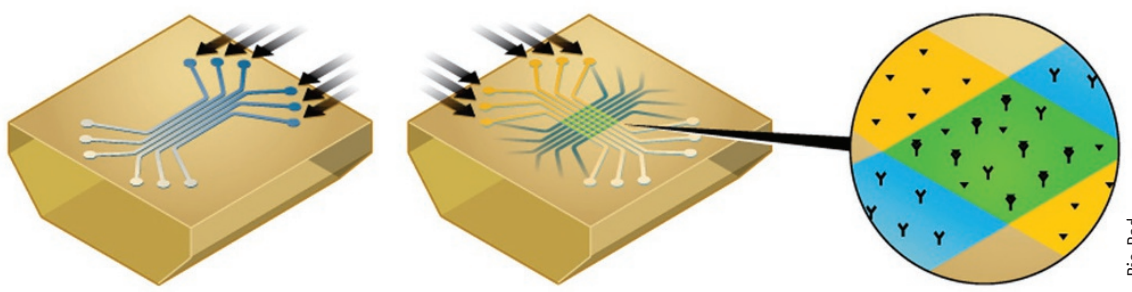

The Prote0n XPR36 protein interaction array system uses a crisscross microfluidic array for the analysis of 36 simultaneous biomolecular interactions. Image inside circle illustrates interaction points.

sitive than conventional non-SPR-based assays, an issue that they traced down to the antibody attachment chemistry.

Corn is also working to improve SPR sensitivity for flat arrays using a new diffraction approach. "Diffraction is great because it sends light out in a new direction so there is low background," says
Corn. But the challenge is to develop surface-sensitive diffraction elements that work in an array format. Here Corn is exploring nanowires and nanorod arrays while other groups are looking at the potential of nanoholes. "SPR is very sensitive to changes in the refractive index of nanogold surfaces," notes Corn. "We have to find the right combination of plasmonic materials that will allow us to do that in a diffraction format."

As SPR sensing increases in throughput and sensitivity, the next big step in its development might not come from a technical innovation, but rather a change in perception as more proteomics researchers finally see the potential of this technique.

1. Haes, A.J. \& Van Duyne, R.P. A nanoscale optical biosensor: sensitivity and selectivity of an approach based on localized surface plasmon resonance spectroscopy of triangular silver nanoparticles. J. Am. Chem. Soc. 124, 10596-10604 (2002).

Nathan Blow is the technology editor for Nature and Nature Methods (n.blow@ boston.nature.com) 
SUPPLIERS GUIDE: COMPANIES OFFERING SURFACE PLASMON RESONANCE AND PROTEOMICS INSTRUMENTS AND ACCESSORIES

\section{Company}

Adron Systems LLC

Agilent

Alcott Chromotography

Analytica of Branford, Inc.

Applied Biosystems

Astra Analytical

Autolab

Bergmann Messgeräte Entwicklung KG

Biaffin

Biosensing Instrument

BioTrove

Bio-Rad

Bruker Daltonics

Comstock Incorporated

De Tech

Evans Analytical Group

Ferran Scientific

Full Spectrum Analytics

Fujifilm Life Science

GB Scientific

GE Healthcare

GWC Technologies

Hitachi Instruments

Horiba Scientific

HT Laboratories

Hybrigenics

In Process Instruments

Invitrogen

Jeol

Jordan TOF Products, Inc.

Kore Technology

Kinomics GmbH

Kratos Analytical

LEAP Technologies

LECO Corp.

M-Scan

Metabolic Solutions, Inc.

Monitor Instruments

mSPEC Group

Omega Engineering, Inc.

Pariss

PerkinElmer

Photonis

ProLab Resources

Reichert

Restek

Scientific Instrument Services, Inc.

SensiQ

Shimadzu Scientific Instruments

Sigma Aldrich

SGE Analytical Sciences

Thermo Fisher Scientific

Varian

Waters
Web address

http://www.adronsystems.com/

http://www.agilent.com/

http://www.alcottchromatography.com/

http://www.aob.com/

http://www.appliedbiosystems.com/

http://astraanalytical.com/

http://www.autolab-instruments.com/

http://www.bme-bergmann.de/

http://www.biaffin.com/

http://www.biosensingusa.com/

http://www.biotrove.com/

http://www.bio-rad.com/

http://www.bdal.com/

http://www.comstockinc.com/

http://www.detechinc.com/

http://www.eaglabs.com/

http://www.ferran.com/

http://www.fullspectrum-inc.com/

http://www.fujifilmlifescienceusa.com/

http://www.gbscientific.com/

http://www.gehealthcare.com/

http://www.gwctechnologies.com/

http://www.hitachi.com/

http://www.horiba.com/scientific/

http://www.ht-labs.com/

http://www.hybrigenics.com/

http://www.in-process.com/

http://www.invitrogen.com/

http://www.jeol.com/

http://www.rmjordan.com/

http://www.kore.co.uk/

http://www.kinomics.com/

http://www.kratos.com/

http://www.leaptec.com/

http://www.leco.com/

http://www.m-scan.com/

http://www.metsol.com/

http://www.monitorinstruments.com/

http://www.mspecgroup.com/

http://www.omega.com/

http://www.nanoparticleanalysis.com/

http://www.perkinelmer.com/

http://www.photonis.com/

http://www.prolabresources.com/

http://www.reichertspr.com/

http://www.restek.com/

http://www.sisweb.com/

http://www.discoversensiq.com/

http://www.shimadzu.com/

http://www.sigmaaldrich.com/

http://www.sge.com/

http://www.thermo.com/

http://www.varianinc.com/

http://www.waters.com/ 